\title{
A systematic literature review of time to return to work and narcotic use after lumbar spinal fusion using minimal invasive and open surgery techniques
}

Xuan Wang ${ }^{1 *}$ (D, Benny Borgman², Simona Vertuani ${ }^{1}$ and Jonas Nilsson ${ }^{1}$

\begin{abstract}
Background: Chronic low back pain is a common health problem for adult workers and causes an enormous economic burden. With the improvement of minimally invasive surgical techniques (MIS) in spinal fusion and the development of fusion devices, more lumbar operations are today being performed through a less invasive technique. When compared with open surgeries (OS), MIS has demonstrated better clinical outcomes including operation time, blood loss, complication rates and length of hospital stay. The aim of this review was to identify and summarize evidence on the time to return to work and the duration of post-operation narcotic use for patients who had lumbar spinal fusion operations using MIS and OS techniques.
\end{abstract}

Methods: A systematic literature review was performed including studies identified from PubMed, EMBASE, the Cochrane Collaboration, and the Centre for Review and Dissemination (CRD) (January 2004-April 2014) for publications reporting on time to return to work and post-operation narcotic use after MIS or OS lumbar spinal fusion surgeries.

Results: Out of a total of 36 included studies, 28 reported on the time to return to work and 17 on the narcotic use after MIS or OS. Four studies described the time to return to work directly comparing MIS and OS. Three studies, from the US, directly compared the duration of narcotic use between MIS- transforaminal lumbar interbody fusion (TLIF) and OS-TLIF. In addition to the time to return to work, 23 studies reported on the rate of return to work and the employment rate before and after surgery, and two Swedish studies presented sick leave data.

Conclusions: There is a gap of good quality data describing the time to return to work and narcotic use after lumbar spinal fusion operations using MIS or OS techniques. However, the current systematic literature review indicates that patients who have lumbar spinal fusion operations, with the MIS procedure, generally return to work after surgery more quickly and require less post-operation narcotics for pain control compared to patients who have OS.

Keywords: Lumbar spinal fusion, Minimal invasive surgery, Open surgery, Transforaminal lumbar interbody fusion, Posterolateral fusion, Posterior lumbar interbody fusion, Anterior lumbar interbody fusion, Systematic literature review, Return to work, Narcotic use

\footnotetext{
* Correspondence: xwang@mapigroup.com

${ }^{1}$ Mapi Group, Klarabergsviadukten 90B, SE-111 64 Stockholm, Sweden

Full list of author information is available at the end of the article
} 


\section{Background}

Chronic low back pain is a leading common health problem for adult workers worldwide [1, 2]; it is the leading cause of activity limitation, job-related disability and absence from work, and it causes an enormous economic burden [3].

The Global Burden of Disease study [3] estimated that low back pain causes more global disability than any other condition. According to its estimates in 2010, the total costs of the condition in the United States exceed \$100 billion annually, with two-thirds of these costs come from lost wages and decreased work productivity [4, 5].

Surgical treatment of the lumbar spine has been shown to be effective in reducing patient's pain and improving function and disability relative to non-surgical treatment [6]. In addition, surgical treatment has been proven to be cost-effective over a 4-year period compared with none-surgical care [7].

A recent study examined the effects of lumbar spinal surgery on work productivity with regard to earnings and absence from work and concluded that reduced productivity losses, after disc herniation surgery, may offset the increased direct medical costs associated with surgery [8]. Lumbar spinal fusion surgery is a viable treatment option for reducing pain and improving function in patients with chronic pain refractory to non-surgical care [9].

Minimally invasive surgery (MIS) techniques for lumbar spinal fusion are equivalent to traditional open surgery (OS) procedures in terms of post-operation fusion rates [10], while MIS has the advantage of reducing tissue damage to the spinal muscles compared to OS [11].

The use of MIS techniques in lumbar spinal surgery has increased as improved patient outcomes and lower hospital costs have been recognized [12-14].

Several studies have demonstrated short term benefits of MIS such as rapid mobilization, shorter length of hospital stay, reduced blood loss, less post-operation pain, reduced risk of infection, and reduced need for postoperation analgesics [15]. Better clinical outcomes compared to OS have been described for a number of different incision approaches such as transforaminal lumbar interbody fusion (TLIF), posterior lumbar interbody fusion (PLIF) and anterior lumbar interbody fusion (ALIF) [16, 17].

Parker et al. showed that MIS-TLIF was associated with reduced costs over 2 years with similar health utilities as OS-TLIF [18] and, MIS-TLIF lumbar spinal fusion resulted in a statistically significant reduction in total hospital costs [14]. However, the economic evaluations of MIS and OS paid little attention to the societal perspective related to initial return to work and productivity after MIS surgery. In addition, earlier narcotic independence following lumbar spinal fusion is another factor that may influence the ability to return to normal work activities.

This systematic literature review (SLR) focused on identifying evidence from published literature on time to return to work and post-operation narcotic use after lumbar spinal fusion operations using MIS or OS techniques. The main objective with the study was to determine whether there is evidence supporting the hypothesis that early post-operation benefits of MIS, compared to OS, have an effect on work productivity.

\section{Methods}

A literature review protocol was developed to design the systematic review detailed search strategies, criteria for study selection, and outcomes to be reported. The PRISMA 2009 checklist (Preferred Items for Systematic Reviews and Meta-Analyses) was used for reporting this review.

\section{Data sources}

The following electronic databases were searched from January 1, 2004 to April 22, 2014: National Library of Medicine's online PubMed, EMBASE, the Cochrane Collaboration, and the Centre for Review and Dissemination (CRD). The search strategy for PubMed is available as Supplementary material (Additional file 1: Sample search strategy). In addition, evidence was obtained online from the following HTA agencies:

- National Institute for Health and Clinical Excellence (NICE)

- Pharmaceutical Benefits Advisory Committee (PBAC)

- Canadian Agency for Drugs and Technologies in Health (CADTH)

Conference proceedings from meetings organized by the following associations were also searched:

- International Society for Pharmacoeconomics Outcomes Research (ISPOR)

- European Federation of National Associations of Orthopaedics and Traumatology (EFORT)

- American Academy of Orthopaedic Surgeons (AAOS)

- Orthopaedic Trauma Association (OTA)

- North American Spine Society (NASS)

- International Society for the Advancement of Spine Surgery (ISASS)

- Society for Minimally Invasive Spine Surgery (SMISS)

A manual review of the bibliographies of identified key publications was also conducted in order to identify any 
relevant publications that were not identified through the electronic database searches.

\section{Study eligibility}

Only studies that fulfilled the inclusion criteria, guided by the PICOS approach [19], were included in the study:

- Published articles, posters, abstracts, reports and conference proceedings

- Studies focused on diseases that need to be treated with lumbar spinal fusion surgery using OS and MIS procedures

- All types of MIS and OS techniques were considered

- Studies of time to return to work, studies on adult population who were not retired and suffering from low back pain and had undergone spinal fusion

- Studies on post-operation narcotic use with no restriction of patients' age

Studies comparing the time to return to work following lumbar spinal fusion with MIS or OS were of primary interest. The following exclusion criteria were used for the retrieved studies:

- Time to return to work studies including patients of age $<18$ or $>65$ years

- Languages other than English, German, and Swedish

- Surgical technique (i.e., MIS or OS) not specified

- Study objective of narcotic use focused on peri-operation rather than post-operation narcotic usage

- Comparisons of different routes of narcotics administration

- Articles published prior to 2004

\section{Study selection}

Studies identified through the searches in PubMed, EMBASE, Cochrane, and CRD were electronically stored in a Reference Manager database (version 12) and entered into an Excel file which facilitated conduction and tracking of selection process. The study selection was completed through two levels of review by two independent reviewers: the Level I review involved scanning of titles and abstracts and the Level II review involved scanning the full text of articles which had not been excluded in the Level I. Any discrepancies between the two reviewers were resolved by a third reviewer.

\section{Data extraction}

Data were extracted on study characteristics, patient information, surgery information, return to work outcomes, narcotic usage, and economic outcomes by a single reviewer. To ensure the validity of the extracted data, studies were randomly quality checked by a second reviewer who did not perform the initial extraction. Any disagreements were resolved through discussion, or by a third party, until consensus was reached.

\section{Quality assessment}

To account for any bias and uncertainty within the publications, included studies were assessed for methodological rigor and quality using the NICE methodology checklist, as set out in the NICE technical manual "Methods for the development of NICE public health guidance (third edition)" [20].

\section{Results}

A total of 1212 unique records were obtained from all the searches; of which, 199 were retrieved from HTA agencies and conference proceedings. Out of these, 236 were selected for full text evaluation and, eventually, 36 studies were included for full text review and dataextraction. The selection process and the number of articles included at each consecutive step of the SLR are described in Fig. 1. The complete list of the included articles is available as supplementary material (Additional file 2: List of included studies).

\section{Characteristics of included studies}

Studies were undertaken in a range of 13 countries, where the majority was found from the US $(n=16)$ followed by South Korea $(n=5)$ and Sweden $(n=3)$. Among the 36 included studies, 28 reported outcomes on the time to return to work and 17 on the use of narcotics after MIS or OS. Out of the 28 studies reporting time to return to work, more than half $(n=18)$ were observational studies, four were randomized controlled trials (RCTs), and five were economic evaluation studies. Only five studies directly compared the time to return to work between MIS and OS and none of them were RCTs. A majority of the studies reporting on post-operation narcotic use were observational studies $(n=13)$ and ten studies directly compared MIS and OS.

Out of the 21 studies reporting on MIS, TLIF was used in 11 studies, followed by ALIF $(n=4)$ and PLIF $(n=2)$. In the studies focusing on OS, TLIF was also found to be the most frequently studied technique $(n=11)$, followed by ALIF $(n=5)$, " $360^{\circ}$ procedure" (i.e., posterolateral fusion plus internal fixation with the variable screw placement device plus interbody fusion) $(n=4)$ and PLIF $(n=4)$. The different surgical procedures performed in the studies included in the review are described in Fig. 2.

Only four studies fulfilled most criteria of the NICE quality assessment checklist $(++)$ and more than half $(n=21)$ only fulfilled a few criteria (Fig. 3$)$. The reason for this is that most observational studies had low representativeness (e.g., a small sample size, single centre 


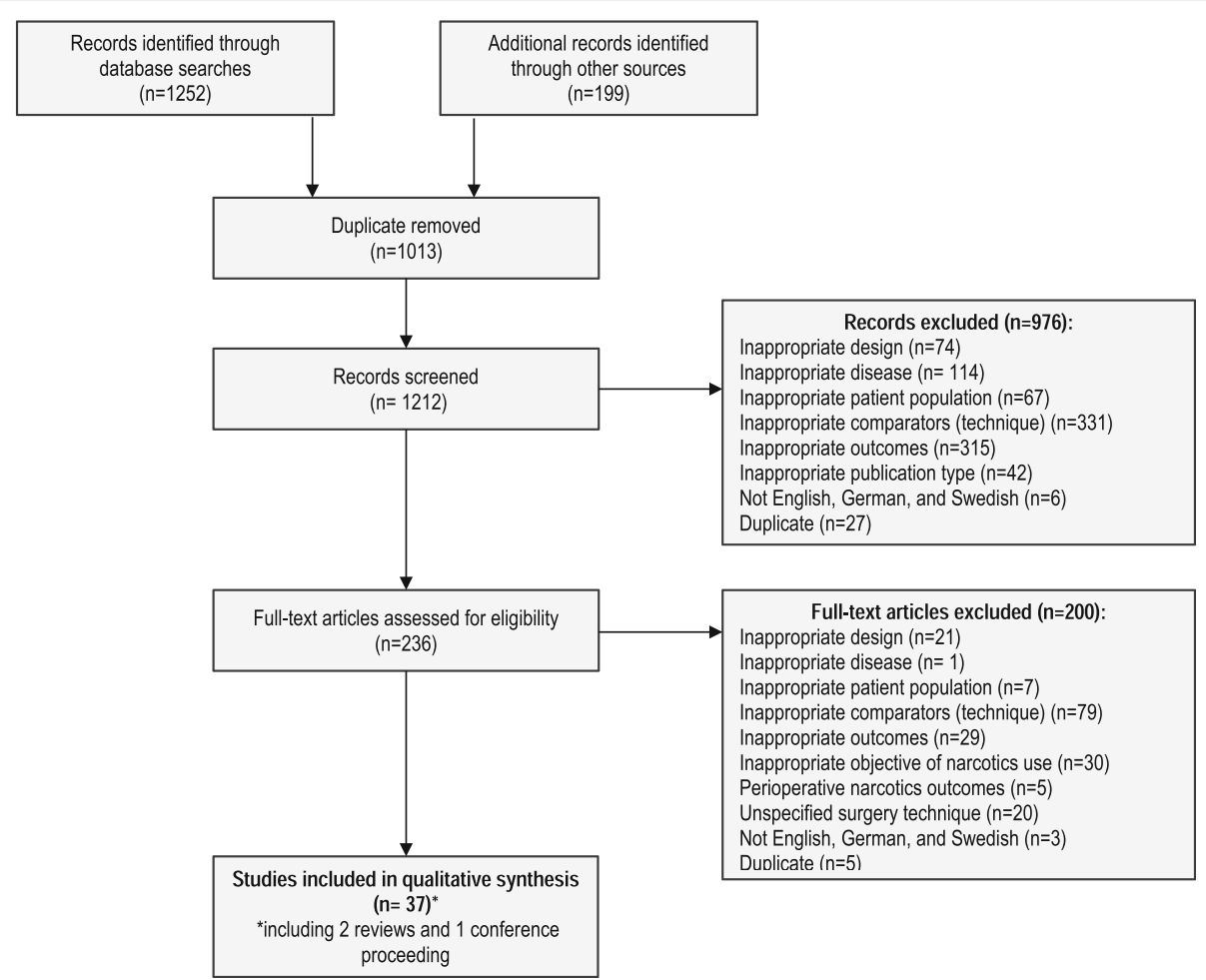

Fig. 1 Flowchart detailing the review process

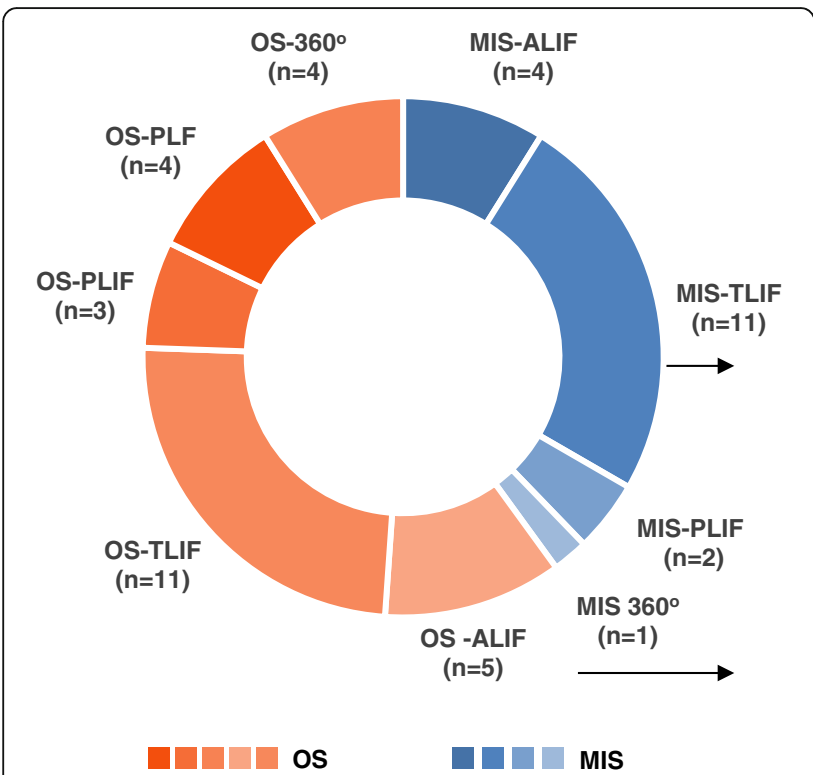

Fig. 2 Surgical procedures performed in the studies included in the review. "360 procedure": Posterolateral fusion plus internal fixation with the variable screw placement device plus interbody fusion; ALIF: Anterior Lumbar Interbody Fusion; MIS: Minimal Invasive Surgery; OS: Open Surgery; PLF: Posterolateral Fusion; PLIF: Posterior Lumbar Interbody Fusion; TLIF: Transforaminal Lumbar Interbody Fusion study, and only one or two surgeons performed the surgeries). In addition, in some cases the study methods and results were not sufficiently described and/or the study questions were not clearly addressed. A table of the assessment results per included article is available as supplementary material (Additional file 3: Quality assessment results using the NICE methodology checklist).

\section{Return to work after lumbar spinal fusion surgery}

The 28 studies including data on return to work reported results differently. Several studies estimated the actual time (i.e., the number of weeks or months) to return to work after surgery, while most studies reported the proportion of patients returning to work at different follow-up intervals after surgery. The relevant studies have been divided into three groups: i) studies that directly compared MIS and OS; ii) studies that used MIS; or iii) OS techniques (Table 1).

\section{Time to return to work}

\section{MIS vs. OS studies}

Four studies $[18,21-23]$ were identified that directly compared the time to return to work after lumbar spinal fusion with the MIS and OS procedures. Out of these, three $[18,21,22]$ have been conducted in the US by the same research group. Adogwa et al. [21] and Parker et al. [18] 


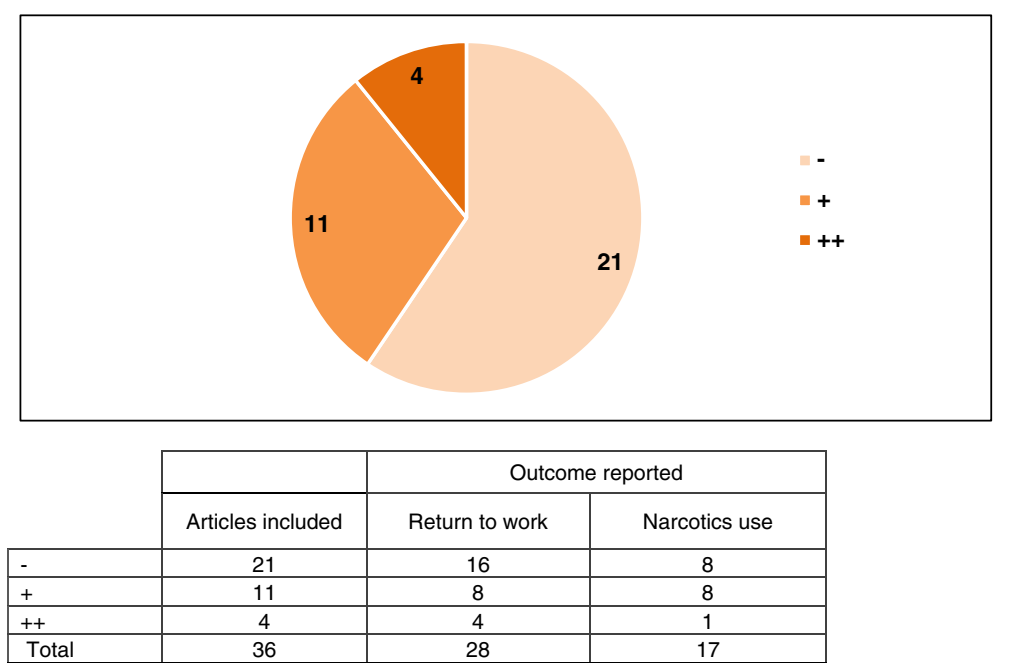

Fig. 3 Quality assessment of the included studies. ++ All or most of the criteria have been fulfilled. Where they have not been fulfilled the conclusions of the study or review are thought very unlikely to alter. + Some of the criteria have been fulfilled. Those criteria that have not been fulfilled or not adequately described are thought unlikely to alter the conclusions. - Few or no criteria fulfilled. The conclusions of the study are thought likely or very likely to alter

reported the time to return to work after of MIS-TLIF or OS-TLIF in a pilot study including 15 patients per group, while Parker et al. [22] studied a larger cohort of 100 patients (50 patients per MIS-TLIF or OS-TLIF group) with a follow-up period of 2 years. This study confirmed the trend from the earlier study [18] where it was observed that patients who had MIS-TLIF returned to work after surgery, significantly earlier compared to patients who had OS-TLIF (7 vs. 11 weeks, $p=0.02$ ).

Kim et al. [23] examined two cohorts of patients who underwent MIS-ALIF or OS-circumferential fusion at two different hospitals. The mean time to return to work after surgery was 3.7 months for patients in the MISALIF group and 3.6 months in the OS-instrumented circumferential fusion group. The difference was not statistically significant.

\section{MIS studies}

Five studies [24-28] reported results on the time to return to work for only MIS or only OS procedures used, thus not comparing the two techniques side by side. Kim et al. [24] retrospectively analysed clinical data from 48 patients who had instrumented MIS-ALIF and 46 patients who had instrumented MIS-TLIF. The mean follow-up period was more than 2 years for both groups and the mean time to return to work was found to be 6.1 months in the ALIF group and 10.9 months in the TLIF group $(p=0.0188)$.

Rouben et al. [25] retrospectively studied 169 patients who had MIS-TLIF, in which 45 patients had two-level spinal fusion. Compared to the results from Kim et al. [24], Rouben et al. [25] found a much shorter time to return to work after surgery; the mean time for the patients who were working immediately before surgery was 11 weeks (median: 8 weeks), while a slightly longer time to return to work (mean: 17 weeks) was observed for patients with work compensation. In addition, about $91 \%$ of all patients were discharged from hospital within $24 \mathrm{~h}$ after surgery and the longest hospital stay was 3 days. Note that this study was carried out in a single centre in the US where MIS surgery was their primary standard of practice and OS was not often carried out. Kim et al. [24] concluded that, the significant difference in the time to return to work between MIS-ALIF and MIS-TLIF may be due to the difference between the surgical techniques and the mean time to return to work (here it refers to the time to a return to full and unrestricted activity rather than the initial return to work after surgery).

\section{OS studies}

Three studies [26-28] focused on only OS. A randomized controlled investigational device exemption trial conducted by Gornet et al. [28] compared 405 patients treated with lumbar disc arthroplasty with a control group of 172 patients who received OS-ALIF. The median time to return to work was 96 days after OS-ALIF surgery.

A small cohort study by Takahashi et al. [27] reported that 18 of $20(90 \%)$ patients who had worked before OSTLIF surgery returned to work after an average of 3.9 months. The time to return to work for patients in the heavy labour group was longer than patients in the light labour group (5.0 months vs. 3.2 months). 


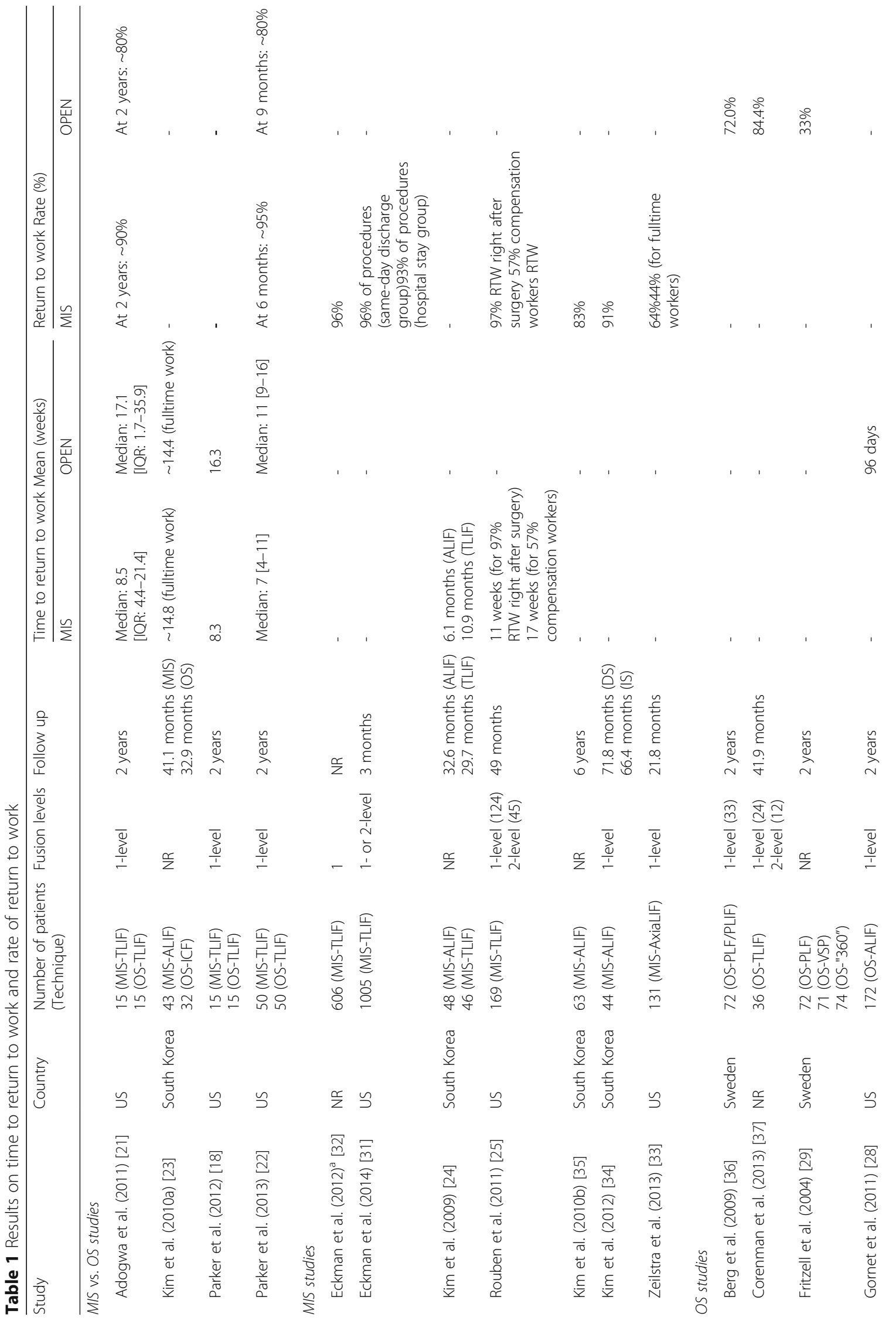




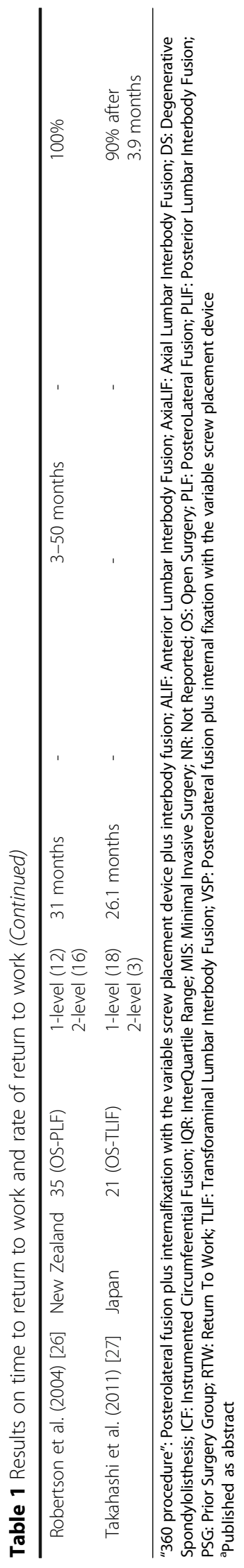


In another study, Robertson et al. [26] investigated 35 patients who had one- or two-level posterolateral fusion (PLF) or PLIF over a 5-year period. Among the 28 patients who were available for follow-up at review, 23 patients were covered by New Zealand's Accident Compensation System and five were not. All five patients with no compensation showed much shorter time to return to work after surgery (average of 3 months, SD not reported) while patients with full compensation had the longest time to return to work (average of 50 months, SD not reported).

\section{Rate of return to work}

A number of studies did not indicate at what time point patients returned to work following surgery; instead, estimates of the rate of return to work were reported. In addition, among the studies only focused on OS, seven compared the employment rate before and after surgery and two studies $[29,30]$ described the number of sick leave days after surgery.

\section{MIS vs. OS studies}

In terms of the rate of return to work, Adogwa et al. [21] and Parker et al. [22] also provided direct comparison between OS-TLIF and MIS-TLIF during 2 years followup. Both studies observed that more patients who were treated with MIS-TLIF returned to work, at each followup time, compared to OS-TLIF patients. Furthermore, more than $90 \%$ of the patients who received MIS returned to work within 12 months and over $80 \%$ of the patients who had OS returned to work within 12 months after surgery.

\section{MIS studies}

Six studies [25, 31-35] reported the rate of return to work for only MIS. In Eckman et al. [31], the same-day discharge cohort consisted of a total of 728 patients who had 808 MIS-TLIF procedures and the hospital stay cohort included 277 patients who had 306 MIS-TLIF procedures. The authors reported that, after 3 months, the rate of return to work were $96 \%$ of procedures in the same-day discharge group and $93 \%$ of procedures in the hospital stay group. In an abstract retrieved from Eckman et al. [32], a total of 394 patients who had MIS-TLIF were working within 30 days prior to surgery and $96 \%$ of patients (367 out of 384 patients with data available) returned to work after surgery, but the follow-up period was not provided.

In Rouben et al. [25], 97\% of patients who worked immediately before surgery and $57 \%$ of patients who had work compensation had returned to work during the 4 years after surgery. Zeilstra et al. [33] retrospectively studied a total of 131 patients who were treated with one-level MIS-axial lumbar interbody fusion (AxiaLIF) with at least 1 year follow-up (mean: $21 \pm 8$ months). The rate of patients who returned to work was not reported. Instead, the authors observed that the employment rate increased from $47 \%$ before surgery to $64 \%$ at final follow-up (pre-operation vs. post-operation: $p<0.001$ ), and the employment rate of full-time work increased from $24 \%$ to $44 \%$ after surgery ( $p$-value not reported). Kim et al. [35] found that $83 \%$ of patients who had MIS-ALIF returned to work within 72 months mean follow-up. Not all patients were able to return to same work or job/level of employment, and about half of them (47.1\%) had to change work or level of job (37.7\%) or even part-time work (9.4\%). A total of 44 patients who had MIS-TLIF were studied by Kim et al. in 2012 [34] and over 90\% of patients who were working before the surgery returned to work within 70 months mean follow-up, and only one patient received work compensation.

Overall, about $83 \%-97 \%$ of patients who were working before surgery were able to return to work within 6 years [25, 31-35]. Although the follow-up intervals varied across these studies, a high rate $(93 \%-96 \%$ of procedures) of patients who returned to work after MIS was observed after the first 3 months [31, 32] and it was not always clear what kind of jobs the patients had. Only Kim et al. [35] reported that a substantial amount of patents had to change work after surgery (47.1\%).

\section{OS studies}

Five studies $[26,27,29,36,37]$ included data on the rate of return to work after OS surgery. Berg et al. [36] reported on a clinical RCT comparing total disc replacement (TDR) and instrumented lumbar spinal fusion (i.e., PLF or PLIF) in Sweden. The clinical outcomes of both groups (TDR: $n=80$ vs. fusion: $n=72$ ) were improved after the operation. After 2 years, $76 \%$ of patients in the fusion group were back to part-time or full-time work.

In another Swedish study, conducted by Fritzell et al. [29], a total of 284 patients were studied who were randomized to either lumbar spinal fusion $(n=217)$ or a non-surgical control group $(n=67)$. Patients who were receiving surgeries were divided into three groups. The authors found that after 2 years: $35 \%$ of the patients who had PLF, 34\% of the patients who had PLF plus internal fixation with the variable screw placement device (VSP) and 39\% of the patients who had PLF plus VSP and interbody fusion ("360 procedure") had returned to work. The difference between the groups may partly be explained by the surgical fusion techniques used.

Corenman et al. [37] presented a small, 2 year retrospective observational study, where data were collected from a return to work questionnaire. The study found that $84.4 \%(27 / 32)$ of the cohort were able to return to the same job as before the surgery. In another small retrospective study with a 1-year minimum follow-up 
period, Takahashi et al. [27] reported that $90 \%$ of the patients (18 of 20) returned to work and 59\% (10 of 17) had to reduce working hours or change work duties. Robertson et al. [26] reported that the number of patients who were on full compensation (i.e., not working) was reduced by $75 \%$ after surgery (pre-operation: $n=16$ vs. post-operation: $n=4$ ).

Overall, the studies focused on OS [26, 27, 29, 36, 37] including PLF, PLIF, and TLIF, as well as circumferential fusion techniques reported a greater variation of rate of return to work (range from 18\% to 100\%) within 3 months to 4 years compared to MIS studies.

Eight of the studies that focused on OS only described the employment rate both pre-operation and postoperation. Overall, a clear trend was observed that the employment rate was increased at 6 months after surgery. Results are summarized in the Supplementary material (Additional file 4: Results of pre-operation vs. postoperation employment rate). In three studies [28, 38, 39] including patients who had ALIF fusion, fairly consistent findings were reported; the employment rate preoperation ranged from $56 \%$ to $58 \%$, while the employment rate 6 months post-operation ranged from $63 \%$ to $73 \%$. In Fritzell et al. [30] and Guyer et al. [40] including patients who received PLF or PLIF lumbar spinal fusion operations, the employment rates were $30 \%$ and $56 \%$ before surgery, and after surgery the employment rates were $72 \%$ and $47 \%$, respectively. It should be noted that the employment rate for patients working part-time was not reported in Guyer et al. [40], while $47 \%$ of the patients worked full-time.

Two Swedish cost-effectiveness studies [29, 30] reported on sick leave for patients who had OS. The number of days on sick leave during a 2-year period after surgery was collected from the Swedish Social Insurance Board. In the first study, Fritzell et al. [29] reported a total of 521 sick leave days per patient who had OS and, in the second study, Fritzell et al. [30] reported a total of 252 sick leave days per patient who had PLF or PLIF during the first 2 years after the operation. Although a relatively high employment rate (i.e., $72 \%$ working full- or part-time) was observed after surgery in Fritzell et al. [30], patients who returned to work after surgery had substantial recurrent sick leave or rehabilitation episodes. The number of levels fused was not reported in either of the studies.

\section{Return to daily activities or full function}

In addition to time and rate of return to work, six studies [23, 24, 34, 41-43] reported the number of patients who returned to full function or normal daily activities after lumbar spinal fusion using MIS or OS during follow-up. Overall, $68 \%-95 \%$ of patients who had MIS returned to daily activities within the mean follow-up of 12 to 70 months after surgery $[24,34,41,42]$ and $58 \%$ of patients return to full function at 2 years in Lee et al. [43], as illustrated in Fig. 4. Higher rates were usually observed at longer follow-up periods. In addition, Kim et al. [23] reported higher rates in return to daily activities for

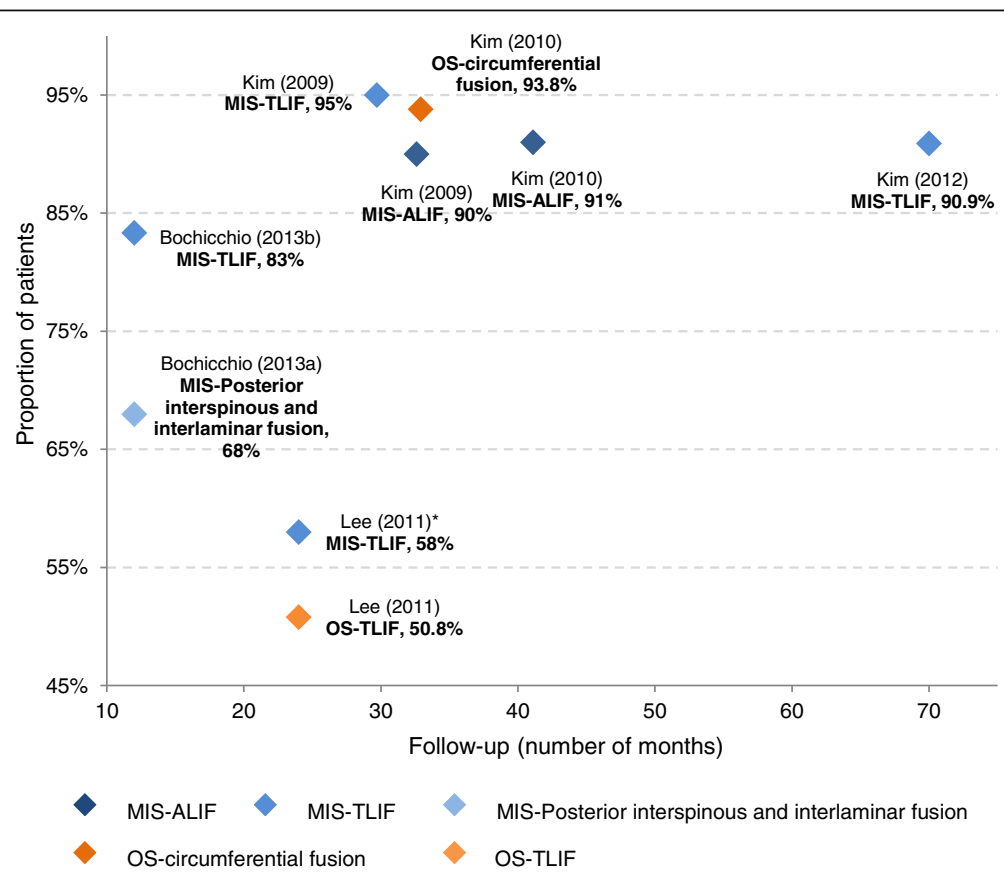

Fig. 4 Proportion of patients return to normal activities or full function. *Results of proportion of patients return to full function in Lee et al. (2011) [43]. ALIF: Anterior Lumbar Interbody Fusion; MIS: Minimally Invasive Surgery; OS: Open Surgery; TLIF: Transforaminal Lumbar Interbody Fusion 
patients who had OS-instrumented circumferential fusion (93.8\%) versus MIS-ALIF (91\%) after about 3 years postoperation, but the difference was not significant.

\section{Post-operation narcotic use after spinal fusion surgery}

Out of 17 studies, 11 directly compared MIS and OS in terms of post-operation narcotic use; however, only three studies $[18,21,22]$ reported on time to narcotic independence and the remaining studies $[18,21,22,25,26$, $28,33,38,43-52]$ presented various results.

\section{Duration of post-operation narcotic use}

Three studies directly compared the time to narcotic independence between MIS and OS (Fig. 5). Adogwa et al. [21] and Parker et al. [18] reported that the length of narcotic use after surgery was, significantly, shorter for patients who underwent MIS-TLIF (median: 2 weeks [21]; mean: 2.6 weeks [18]) compared with OS-TLIF (median: 4 weeks [21]; mean: 6.5 weeks [18]; MIS vs. OS: $p=0.008[18,21]$ ). In Parker et al. [22], patients in the MIS-TLIF group had threefold shorter time on narcotic use, post-operation, than patients in the OS-TLIF group (median: 3 weeks vs. 9 weeks); however, this substantial difference did not reach statistical significance $(p=0.14)$ due to great variability in the post-operation narcotic use for both cohorts.

\section{Other results of post-operation narcotics}

In addition to the duration of post-operation narcotic use, a number of studies reported results reflecting other post-operation narcotic uses (Additional file 5: Other results of post-operation narcotic usage). A total of ten studies [18, 21, 22, 43, 44, 46, 48-51] directly compared the MIS and OS procedures, three studies [25, 33, 52] only focused on MIS and five studies only used OS [26, $28,38,45,47]$. The proportion of patients who used narcotics for pain control, at different follow-up intervals were frequently described, but the definition of narcotics was not clearly described. Although various approaches were used to present the outcomes of post-operation

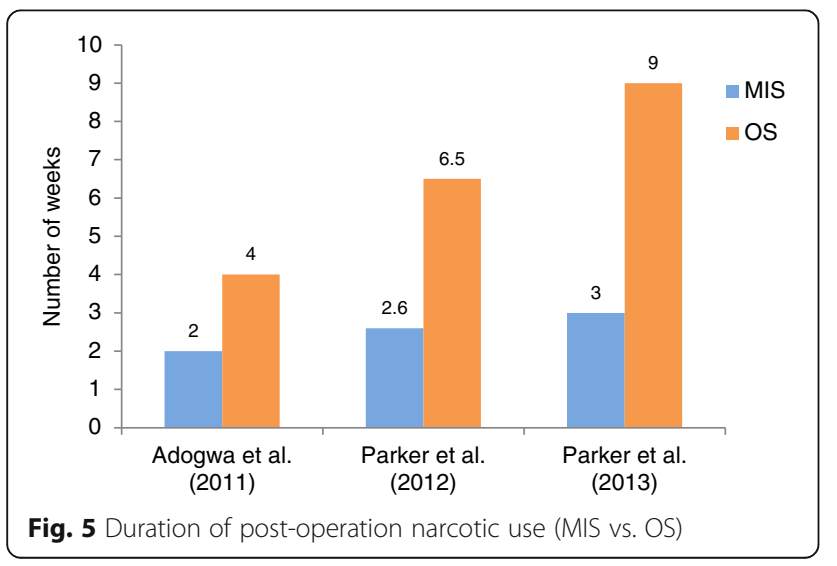

narcotic use, the results were favourable for the MIS technique compared to the OS across all these studies.

\section{Discussion}

With the improvement of MIS surgical techniques in spinal fusion and the development of fusion devices, more operations are today being performed using MIS techniques. A number of previous studies $[16,17]$ have demonstrated that MIS techniques for lumbar spinal fusion have improved the clinical outcomes including operation time, blood loss, complication rates and length of hospital stay, resulting in less hospital resource utilization; however the benefits of MIS techniques such as faster return to work and productivity which means reducing indirect costs to patients and society are less explored. This SLR was conducted to identify and summarize evidence on the time to return to work and the duration of post-operation narcotic use for patients who had lumbar spinal fusion operations using the MIS and OS techniques. Compared with a previous literature review, published by Parker et al. in 2012 [53], this SLR has a broader scope of time to return to work (e.g., time, rate, employment status, and sick leave) and less restrictions on the type of surgical technique that were used (e.g., TLIF or PLIF).

Out of a total of 36 included studies (including five abstracts [32, 41-43, 46]), two thirds of the studies $(n=25)$ were observational studies and only five studies $[28,36,38,40,48]$ were RCTs. This might to some extent reflect the actual situation in surgical research, i.e., retrospective case series are more commonly used, usually with small cohorts, while RCTs are more widely used for pharmacological therapies [54, 55]. TLIF was found to be the most frequently studied technique for lumbar spinal fusion operations regardless if MIS or OS was used. Twenty-seven studies described the position of the fusion or the number of levels that were fused for the study patients; out of which, nine studies [25-27, 31, $32,37,47,56,57]$ included patients who had two or more levels fused (range from 14\% - 57\% of total studied patients), but the results were not presented separately by fusion levels. Therefore, the current SLR mainly provides evidence of the time to return to work and the post-operation narcotic use for patients after a singlelevel lumbar spinal fusion.

The current SLR identified four studies [18, 21-23] that directly compared the time to return to work for patients who had lumbar spinal fusion with the MIS and OS procedures. The three US studies [18, 21, 22] showed almost half the time to return to work for patients who had MIS-TLIF (range of absolute mean: 7.0 to 8.5 weeks) compared to patients who had OS-TLIF (11.0 to 17.1 weeks). Additionally, more than $80 \%$ of the patients who received MIS-TLIF or OS-TLIF returned 
to work within 12 months after surgery [21, 22]. When patients who had MIS-ALIF were compared with OSinstrumented circumferential fusion, Kim et al. [23] reported similar times to return to work for both procedures (3.7 vs. 3.6 months), but no statistically significant difference. It should be noted that two different surgical fusion techniques have been performed at separate hospitals which may explain the lack of difference. Furthermore, the mean follow-up period was 8 months shorter for patients in the OS group compared to the MIS group (32.9 vs. 41.1 months). In this Korean study [23] the surgical techniques were different from the three US studies $[18,21,22]$ and a stringent definition of return to work was applied: "return to full and unrestricted activity", as a result, the time to return to work for both the MIS and the OS groups were different from the results presented in the US studies [18, 21, 22], potentially reflecting cultural practices in Korea. In Kim et al. [24], the time to return to full and unrestricted activity were also used and, therefore, longer times to return to work have been presented compared to the US studies for the MIS groups [18, 21, 22]. Another US study, conducted by Rouben et al. [25], reported a similar time to return to work (11 weeks) as the US studies [18, 21, 22] for patients who had MIS-TLIF. An US study by Gornet et al. [28] focused on only OS-ALIF reported time to return to work with 96 days (13.7 weeks). Based on the findings from these US studies [18, 21, 22, 28], it can be concluded that MIS patients return to work faster compared to OS patients.

The NICE checklist was followed for quality assessment in this review; in general the quality of the included studies was poor. Only four studies [28, 29, 38, 40] fulfilled most criteria and many of the included studies $(n=21)$ only fulfilled few or very few criteria, e.g., the patient samples were small ranging from eight to 72 patients per treatment arm, and were often from a single institution treated by only one or two surgeons. Clearly, there is a gap of evidence around how the time to return to work after lumbar spinal fusion operations differs between the MIS and OS techniques. The interpretation of the current evidence suggests that the time to work after MIS is shorter than after OS operations.

In addition to the time to return to work, several studies were found that reported on the rate of return to work and the employment rate before and after surgery. For patient who had MIS, 83\%-97\% return to work after surgery within 3 months to 6 years. For patients who had OS a greater variation in the share of patient who returned to work was observed ranging from $18 \%$ to $100 \%$ within 3 months to 4 years depending on the techniques being used, i.e., PLF, PLIF, TLIF and circumferential fusion. Information regarding the type of jobs the patients was working with before and after the surgery was limited. Kim et al. [35] and Takahashi et al. [27] reported that a substantial amount of patients had to change work or reduce working hours after surgery (47.1 and 59\%, respectively).

With regard to the employment rate pre- and postoperation, the combined employment rate of full-time and part-time work was frequently reported. Studies focused on OS only in general showed a clear trend of increased employment rate after surgery. Three studies $[28,38,39]$ that focused on OS-ALIF reported fairly consistent time to return to work of 6 months. The employment rate pre-operation ranged from $56 \%$ to $58 \%$, while the employment rate 6 months post-operation ranged from $63 \%$ to $73 \%$. In addition, only two Swedish cost-effectiveness studies $[29,30]$ were found that reported on sick-leave for patients who had lumbar spinal fusion with OS; no information on sick-leave was found for MIS in this SLR.

Six studies [23, 24, 34, 41-43] were found describing the return to normal daily activities or full function post-operation. However, the definition of return to daily activities and full function was, generally, not clearly described.

Various factors may affect the return to work, which have not been widely and consistently assessed across the identified studies. Several studies reported the work status (e.g., full-time or part-time), type of work (e.g., heavy or light labour work) and workers' compensation status prior to surgery led to different return rate or time duration after MIS or OS [26, 27, 29, 37, 38]. In addition, most studies reported patients' clinical characteristics (e.g., blood loss, surgery time, interbody fusion technique and number of fusion levels), which may affect the return to work. Furthermore, surgery-related and neurological complications may be associated with the return to work. However, due to the small sample size and few observed complications, these factors have not been sufficiently studied [22, 43, 49, 51]. Recent reviews, in other conditions $[58,59]$, have reported that age, sex, education and other social-economic factors are important factors affecting the return to work, which could be investigated in future studies on MIS or OS in spinal infusion.

Altogether the results of time to and rate of return to work, and comparisons of pre- and post-operative employment rates show benefit for MIS compared to OS. A review of cost of illness studies on chronic low back pain [60] has showed that the direct cost associated with low back pain accounted for only $22 \%$ of the total costs, which indicated that the indirect cost caused by the loss of productivity contributed with a much larger share of the overall cost for chronic low back pain. The possibility to return to work faster after surgery and be productive may be one of the largest societal advantages of MIS compared 
to OS. Reduced absenteeism from work is not only important for the societal costs but also for patients' quality of life; patients who are able to faster return to normal daily activities are more likely to recover faster due to effective relief of symptoms and disability [61, 62].

Post-operation narcotic use was at focus in 17 studies; out of which 11 studies directly compared MIS versus OS, but again only the three US studies [18, 21, 22] reported the duration of post-operation narcotic use. Similar results as for the time to return to work, MISTLIF was associated with half the period of narcotic use after surgery (range of absolute mean time of postnarcotic use: 2-3 weeks) compared to OS-TLIF (mean range: $3-9$ weeks). The difference was statistically significant in Adogwa et al. [21] and Parker et al. [18] but not in Parker et al. [22] where patients in the OS-TLIF group had threefold longer narcotic use post-operation than MIS-TLIF (median: 3 vs. 9 weeks). It is likely that the advantages of the MIS procedure (e.g., less muscle damage) contributed to the shorter time of narcotic use during the recovery period after surgery. Duration of narcotic use after surgery may be affected by pre-operation narcotic uses, because patients who used narcotics pre-operation are more likely to continue their pain medications after surgery or require time to quit the narcotics due to, for example, rebound effect; however, the duration of preoperation narcotic use was not available in these studies. No significant difference in the use of narcotics postoperation between MIS and OS was found in most studies that directly compared MIS to OS, but a majority of studies indicated less use of narcotics for patients after MIS.

Chronic back pain in the lumber region is one of leading cause of disability and there is a high incidence of psychiatric comorbidities (e.g., depression) [63] and substance abuse (narcotics or other drugs) [64] among patients with degenerative disc disease. None of the studies discussed the psychiatric situation of the patient and the abuse of narcotics among patients with disc degenerative disorders. Furthermore, most studies were observational studies and the decision to carry out OS or MIS spinal fusion operations often depended on surgeon's proficiency and preference.

The post-operation narcotic use was not clearly, and consistently, defined across studies which limit the possibility to compare the results. Just as the time to return work, the narcotic use following spinal surgery operation is a likely differentiator between the MIS and OS procedures. Therefore, future clinical trials would benefit from more focus on the narcotic use post-operation.

\section{Conclusions}

This study shows that there is a gap of good quality data regarding the time to return to work and narcotic use after lumbar spinal fusion operations using MIS or OS techniques. This study also indicates that patients who have lumbar spinal fusion operations with the MIS procedure generally return to work after surgery more quickly and require less post-operation narcotics for pain control compared to patients who had OS. As the societal costs for these patients are likely to be significant, and the availability of studies with high quality is low, it is of great importance for future studies to include data collection regarding time to return to work and narcotic use during the post-operation follow-up period.

\section{Additional files}

Additional file 1: Sample search strategy. The sample search strategy contains all key terms that were searched to identify studies from electronic bibliographic databases. (DOCX 16 kb)

Additional file 2: List of included studies. A list of studies that were finally included after title/abstract and full text evaluations in this SLR. (DOCX $18 \mathrm{~kb}$ )

Additional file 3: Quality assessment results using the NICE methodology checklist. This table presents the quality assessment results per included studies using the NICE methodology checklist [65]. (DOCX 85 kb)

Additional file 4: Results of pre-operation vs. post-operation employment rate. This table summarizes the results of pre-operation vs. post-operation employment rate from the eight studies that focused on OS [65]. (DOCX $31 \mathrm{~kb}$ )

Additional file 5: Other results of post-operation narcotic usage. This table shows the results of post-operation narcotic uses other than the duration of post-operation narcotic use. (DOCX 59 kb)

\section{Abbreviations}

AAOS: American Academy of Orthopaedic Surgeons; ALIF: Anterior lumbar interbody fusion; AxiaLIF: Axial lumbar interbody fusion; CADTH: Canadian Agency for Drugs and Technologies in Health; CDR: Canadian Agency for Drugs; CRD: Centre for Reviews and Dissemination; DARE: Database of abstracts of reviews of effects; EFFOT: European Federation of National Associations of Orthopaedics and Traumatology; ISASS: International Society for the Advancement of Spine Surgery; ISPOR: International Society for Pharmacoeconomics Outcomes Research; MIS: Minimally invasive surgery; NASS: North American Spine Society; NHS EED: NHS Economic Evaluations Database; NICE: National Institute and Clinical Excellence; NIH: National Institutes of Health; NSAID: Non-Steroidal Anti-Inflammatory Drug; OS: Open surgery; OTA: Orthopaedic Trauma Association; PBAC: Pharmaceutical Benefits Advisory Committee; PLF: Posterolateral fusion; PLIF: Posterior lumbar interbody fusion; RCT: Randomized controlled trial; SMISS: Society for MINIMALLY INVASIVE SPINE SURGERY; TDR: Total disc replacement;

TLIF: Transforaminal lumbar interbody fusion

\section{Acknowledgements}

Not applicable.

\section{Funding}

This study was funded by Medtronic. BB is a paid employee of Medtronic. XW, JN and SV were paid consultants to Medtronic.

\section{Availability of data and materials}

This study does not contain any data but the data abstraction sheet is available from the corresponding author upon request.

\section{Authors' contributions}

$X W, B B, S V$ and JN contributed equally to the design of the study methodology. XW, JN and SV conducted the literature review. XW, BB, SV and JN contributed to the analysis and interpretation of the results. XW, BB, SV and JN reviewed draft versions of the manuscript and approved the final version. 


\section{Ethics approval and consent to participate}

No ethics approval was necessary for the current study.

\section{Consent for publication}

Not applicable.

\section{Competing interests}

The authors declare that they have no competing interests.

\section{Publisher's note}

Springer Nature remains neutral with regard to jurisdictional claims in published maps and institutional affiliations.

\section{Author details}

'Mapi Group, Klarabergsviadukten 90B, SE-111 64 Stockholm, Sweden. ${ }^{2}$ Spine \& Biologics Medtronic International Trading SARL, Tolochenaz, Switzerland.

Received: 19 August 2015 Accepted: 19 June 2017

Published online: 27 June 2017

\section{References}

1. Andersson GB. Epidemiological features of chronic low-back pain. Lancet. 1999:354(9178):581-5.

2. Dionne CE, Dunn KM, Croft PR. Does back pain prevalence really decrease with increasing age? A systematic review. Age Ageing. 2006;35(3):229-34.

3. Hoy D, March L, Brooks P, Blyth F, Woolf A, Bain C, et al. The global burden of low back pain: estimates from the global burden of disease 2010 study. Ann Rheum Dis. 2014;73(6):968-74.

4. Guo HR, Tanaka S, Halperin WE, Cameron LL. Back pain prevalence in US industry and estimates of lost workdays. Am J Public Health. 1999;89(7): 1029-35.

5. Katz JN. Lumbar disc disorders and low-back pain: socioeconomic factors and consequences. J Bone Joint Surg Am. 2006;88(Suppl 2):21-4.

6. Weinstein JN, Tosteson TD, Lurie JD, Tosteson AN, Blood E, Hanscom B, et al. Surgical versus nonsurgical therapy for lumbar spinal stenosis. N Engl J Med. 2008:358(8):794-810.

7. Tosteson AN, Tosteson TD, Lurie JD, Abdu W, Herkowitz H, Andersson G, et al. Comparative effectiveness evidence from the spine patient outcomes research trial: surgical versus nonoperative care for spinal stenosis, degenerative spondylolisthesis, and intervertebral disc herniation. Spine (Phila Pa 1976). 2011;36(24):2061-8.

8. Koenig L, Dall TM, Gu Q, Saavoss J, Schafer MF. How does accounting for worker productivity affect the measured cost-effectiveness of lumbar discectomy? Clin Orthop Relat Res. 2014;472(4):1069-79.

9. Phillips FM, Slosar PJ, Youssef JA, Andersson G, Papatheofanis F. Lumbar spine fusion for chronic low back pain due to degenerative disc disease: a systematic review. Spine (Phila Pa 1976). 2013:38(7):E409-22.

10. Wu RH, Fraser JF, Hartl R. Minimal access versus open transforaminal lumbar interbody fusion: meta-analysis of fusion rates. Spine (Phila Pa 1976). 2010; 35(26):2273-81.

11. Skovrlj B, Gilligan J, Cutler HS, Qureshi SA. Minimally invasive procedures on the lumbar spine. World J Clin Cases. 2015;3(1):1-9.

12. Peng CW, Yue WM, Poh SY, Yeo W, Tan SB. Clinical and radiological outcomes of minimally invasive versus open transforaminal lumbar interbody fusion. Spine (Phila Pa 1976). 2009:34(13):1385-9.

13. Wang MY, Cummock MD, Yu Y, Trivedi RA. An analysis of the differences in the acute hospitalization charges following minimally invasive versus open posterior lumbar interbody fusion. J Neurosurg Spine. 2010;12(6):694-9.

14. Wang MY, Lerner J, Lesko J, McGirt MJ. Acute hospital costs after minimally invasive versus open lumbar interbody fusion: data from a US national database with 6106 patients. J Spinal Disord Tech. 2012;25(6):324-8.

15. Tian NF, Wu YS, Zhang XL, Xu HZ, Chi YL, Mao FM. Minimally invasive versus open transforaminal lumbar interbody fusion: a meta-analysis based on the current evidence. Eur Spine J. 2013;22(8):1741-9.

16. Goldstein $\mathrm{CL}$, Macwan K, Sundararajan K, Rampersaud YR. Comparative outcomes of minimally invasive surgery for posterior lumbar fusion: a systematic review. Clin Orthop Relat Res. 2014;472(6):1727-37.

17. Sidhu GS, Henkelman E, Vaccaro AR, Albert TJ, Hilibrand A, Anderson DG, et al. Minimally invasive versus open posterior lumbar interbody fusion: a systematic review. Clin Orthop Relat Res. 2014;472(6):1792-9.
18. Parker SL, Adogwa O, Bydon A, Cheng J, McGirt MJ. Cost-effectiveness of minimally invasive versus open transforaminal lumbar interbody fusion for degenerative spondylolisthesis associated low-back and leg pain over two years. World Neurosurg. 2012:78(1-2):178-84.

19. Higgins JP, Green S: Cochrane handbook for systematic reviews of interventions version 5.0. 2 [updated September 2009]. The Cochrane Collaboration, 2009. Available from www.cochrane-handbook.org 2010.

20. Methods for the development of NICE public health guidance (third edition). Appendix F Quality appraisal checklist - quantitative intervention studies. In.: National Institute for Health and Clinical Evidence (NICE) 2012. https://www.nice.org.uk/process/pmg4/chapter/introduction.

21. Adogwa O, Parker SL, Bydon A, Cheng J, McGirt MJ. Comparative effectiveness of minimally invasive versus open transforaminal lumbar interbody fusion: 2-year assessment of narcotic use, return to work, disability, and quality of life. J Spinal Disord Tech. 2011;24(8):479-84.

22. Parker SL, Mendenhall SK, Shau DN, Zuckerman SL, Godil SS, Cheng JS, et al. Minimally invasive versus open Transforaminal lumbar Interbody fusion for degenerative Spondylolisthesis: Comparative effectiveness and cost-utility analysis. World Neurosurg. 2013;

23. Kim J-S, Kim D-H, Lee S-H, Park C-K, Hwang J-H, Cheh G, et al. Comparison study of the instrumented circumferential fusion with instrumented anterior lumbar interbody fusion as a surgical procedure for adult low-grade isthmic spondylolisthesis. In: World Neurosurgery. Vol. 73. 5th ed. New York: Elsevier Inc.; 2010. p. 565-71.

24. Kim JS, Kang BU, Lee SH, Jung B, Choi YG, Jeon SH, et al. Mini-transforaminal lumbar interbody fusion versus anterior lumbar interbody fusion augmented by percutaneous pedicle screw fixation: a comparison of surgical outcomes in adult low-grade isthmic spondylolisthesis. J Spinal Disord Tech. 2009;22(2):114-21.

25. Rouben D, Casnellie M, Ferguson M. Long-term durability of minimal invasive posterior transforaminal lumbar interbody fusion: a clinical and radiographic follow-up. J Spinal Disord Tech. 2011;24(5):288-96.

26. Robertson PA, Jackson SA. Prospective assessment of outcomes improvement following fusion for low back pain. J Spinal Disord Tech. 2004;17(3):183-8.

27. Takahashi T, Hanakita J, Minami M, Honda F, Kuraishi K. Surgical outcome and postoperative work status of lumbar discogenic pain following transforaminal interbody fusion. Neurol Med Chir (Tokyo). 2011;51(2):101-7.

28. Gornet MF, Burkus JK, Dryer RF, Peloza JH. Lumbar disc arthroplasty with maverick disc versus stand-alone interbody fusion: a prospective, randomized, controlled, multicenter investigational device exemption trial. Spine (Phila Pa 1976). 2011;36(25):E1600-11.

29. Fritzell $P$, Hagg O, Jonsson D, Nordwall A. Cost-effectiveness of lumbar fusion and nonsurgical treatment for chronic low back pain in the Swedish lumbar spine study: a multicenter, randomized, controlled trial from the Swedish lumbar spine study group. Spine (Phila Pa 1976). 2004;29(4):421-34.

30. Fritzell $P$, Berg S, Borgstrom F, Tullberg T, Tropp H. Cost effectiveness of disc prosthesis versus lumbar fusion in patients with chronic low back pain: randomized controlled trial with 2-year follow-up. Eur Spine J. 2011;20(7): 1001-11.

31. Eckman WW, Hester L, McMillen M. Same-day discharge after minimally invasive transforaminal lumbar interbody fusion: a series of 808 cases. Clin Orthop Relat Res. 2014;472(6):1806-12.

32. Eckman WW, Hester LG, McMillen M. Unilateral minimally invasive Transforaminal lumbar Interbody fusion (MITLIF): results of 670 cases discharged the day of surgery. The Spine Journal. 2012;12(9):S119.

33. Zeilstra DJ, Miller LE, Block JE. Axial lumbar interbody fusion: a 6-year single-center experience. In: Clinical interventions in aging. Vol. 8. Auckland: Dove Medical Press Ltd.; 2013. p. 1063-9.

34. Kim JS, Jung B, Lee SH. Instrumented Minimally Invasive spinal-Transforaminal Lumbar Interbody Fusion (MIS-TLIF); Minimum 5-years Follow-up With Clinical and Radiologic Outcomes. J Spinal Disord Tech. 2012. https://www.ncbi.nlm. nih.gov/pubmed/?term=23027364.

35. Kim J-S, Choi WG, Lee S-H. Minimally invasive anterior lumbar interbody fusion followed by percutaneous pedicle screw fixation for isthmic spondylolisthesis: minimum 5-year follow-up. In: Spine Journal. Vol. 10. 5th ed. New York: Elsevier Inc.; 2010. p. 404-9.

36. Berg S, Tullberg T, Branth B, Olerud C, Tropp H. Total disc replacement compared to lumbar fusion: a randomised controlled trial with 2-year follow-up. Eur Spine J. 2009:18(10):1512-9.

37. Corenman DS, Gillard DM, Dornan GJ, Strauch EL. Recombinant human bone morphogenetic protein-2-augmented transforaminal lumbar interbody fusion for the treatment of chronic low back pain secondary to 
the homogeneous diagnosis of discogenic pain syndrome: two-year outcomes. Spine (Phila Pa 1976). 2013;38(20):E1269-77.

38. Blumenthal S, McAfee PC, Guyer RD, Hochschuler SH, Geisler FH, Holt RT, et al. A prospective, randomized, multicenter Food and Drug Administration investigational device exemptions study of lumbar total disc replacement with the CHARITE artificial disc versus lumbar fusion: part l: evaluation of clinical outcomes. Spine (Phila Pa 1976). 2005;30(14):1565-75.

39. Fayssoux R, Goldfarb NI, Vaccaro AR, Harrop J. Indirect costs associated with surgery for low back pain-a secondary analysis of clinical trial data. Popul Health Manag. 2010;13(1):9-13.

40. Guyer RD, McAfee PC, Banco RJ, Bitan FD, Cappuccino A, Geisler FH, et al. Prospective, randomized, multicenter Food and Drug Administration investigational device exemption study of lumbar total disc replacement with the CHARITE artificial disc versus lumbar fusion: five-year follow-up. Spine J. 2009;9(5):374-86.

41. Bochicchio M, Frati A, Rea G, Familiari P, Raco A, Nardi PV. Minimally invasive posterior interspinous and interlaminar fusion device for one level fusion in degenerative lumbar disease: Mid term clinical, and radiological evaluation and complications report. European Spine Journal. 2013;22(4):932.

42. Bochicchio M, Bakaloudis G, Astolfi S, Nardi PV. Minimally invasive technique with transforaminal interbody fusion (TLIF) and interspinous/interlaminar arthrodesis (IFD). Mid term radiologic and clinical evaluation: Fusion rate? Complications? Real advantages? European Spine Journal. 2013;22(4):907.

43. Lee KH, Yue WM, Yeo W, Soeharno H, Tan SB. Clinical and radiological outcomes of open versus minimally invasive transforminal lumbar interbody fusion. The Spine Journal. 2011;11(10):S102-S103.

44. Buttermann GR, Thorson TM, Mullin WJ. Outcomes of posterior facet versus pedicle screw fixation of circumferential fusion: a cohort study. In: European spine Journal. Vol. 23. 2nd ed. Heidelberg: Springer Verlag; 2014. p. 347-55.

45. Froholdt A, Reikeraas O, Holm I, Keller A, Brox Jl. No difference in 9-year outcome in CLBP patients randomized to lumbar fusion versus cognitive intervention and exercises. Eur Spine J. 2012;21(12):2531-8.

46. Hamid N, Roy DD, Vemaraju R, Furtado NR, Abbas N. Percutaneous spinal stabilization-is the future here? Eur Spine J. 2013;22(Suppl 1):S64. doi:10.1007/s00586-013-2664-1.

47. Potter BK, et al. Transforaminal lumbar interbody fusion: clinical and radiographic results and complications in 100 consecutive patients. J Spinal Disord Tech. 2005;18(4):337-46.

48. Rodriguez-Vela J, Lobo-Escolar A, Joven-Aliaga E, Herrera A, Vicente J, Sunen $E$, et al. Perioperative and short-term advantages of mini-open approach for lumbar spinal fusion. In: European spine Journal. Vol. 18. 8th ed. Heidelberg: Springer Verlag; 2009. p. 1194-201.

49. Isaacs RE, Podichetty VK, Santiago P, Sandhu FA, Spears J, Kelly K, et al. Minimally invasive microendoscopy-assisted transforaminal lumbar interbody fusion with instrumentation. J Neurosurg Spine. 2005;3(2):98-105.

50. Cheng JS, Park P, Le H, Reisner L, Chou D, Mummaneni PV. Short-term and long-term outcomes of minimally invasive and open transforaminal lumbar interbody fusions: is there a difference? Neurosurg Focus. 2013;35(2):E6.

51. Kim DY, Lee SH, Chung SK, Lee HY. Comparison of multifidus muscle atrophy and trunk extension muscle strength: percutaneous versus open pedicle screw fixation. Spine (Phila Pa 1976). 2005;30(1):123-9.

52. Schwender JD, Holly LT, Rouben DP, Foley KT. Minimally invasive transforaminal lumbar interbody fusion (TLIF): technical feasibility and initial results. J Spinal Disord. Tech. 2005;18(Suppl):S1-6.

53. Parker SL, Lerner J, McGirt MJ. Effect of minimally invasive technique on return to work and narcotic use following transforaminal lumbar inter-body fusion: a review. Prof Case Manag. 2012;17(5):229-35.

54. Cook JA. The challenges faced in the design, conduct and analysis of surgical randomised controlled trials. Trials. 2009;10:9.

55. Macefield RC, Boulind CE, Blazeby JM. Selecting and measuring optimal outcomes for randomised controlled trials in surgery. Langenbeck's Arch Surg. 2014;399(3):263-72.

56. Brotis AG, Paterakis KN, Tsiamalou PM, Fountas KN, Hahjigeorgiou GM, Karavelis A. Instrumented posterior lumbar fusion outcomes for lumbar degenerative disorders in a southern European, semirural population. J Spinal Disord Tech. 2010;23(7):444-50.

57. Gillard DM, Corenman DS, Dornan GJ. Failed less invasive lumbar spine surgery as a predictor of subsequent fusion outcomes. Int Orthop. 2014; 38(4):811-5.
58. Shi Q, Sinden K, MacDermid JC, Walton D, Grewal R. A systematic review of prognostic factors for return to work following work-related traumatic hand injury. J Hand Ther. 2014;27(1):55-62. quiz 62

59. Kleim BD, Malviya A, Rushton S, Bardgett M, Deehan DJ. Understanding the patient-reported factors determining time taken to return to work after hip and knee arthroplasty. Knee Surg Sports Traumatol Arthrosc. 2015;23(12):3646-52.

60. Dagenais S, Caro J, Haldeman S. A systematic review of low back pain cost of illness studies in the United States and internationally. Spine J. 2008;8(1):8-20.

61. Mokhtar SA, McCombe PF, Williamson OD, Morgan MK, White GJ, Sears WR. Health-related quality of life: a comparison of outcomes after lumbar fusion for degenerative spondylolisthesis with large joint replacement surgery and population norms. Spine J. 2010;10(4):306-12.

62. Pekkanen $L$, Neva MH, Kautiainen $H$, Dekker J, Piitulainen $K$, Wahlman M, et al. Disability and health-related quality of life in patients undergoing spinal fusion: a comparison with a general population sample. BMC Musculoskelet Disord. 2013;14:211

63. Reme SE, Tangen T, Moe T, Eriksen HR. Prevalence of psychiatric disorders in sick listed chronic low back pain patients. Eur J Pain. 2011;15(10):1075-80.

64. Martell BA, O'Connor PG, Kerns RD, Becker WC, Morales KH, Kosten TR, et al. Systematic review: opioid treatment for chronic back pain: prevalence, efficacy, and association with addiction. Ann Intern Med. 2007;146(2):116-27.

65. Wenger M, Sapio N, Markwalder TM. Long-term outcome in 132 consecutive patients after posterior internal fixation and fusion for grade I and II isthmic spondylolisthesis. In: Journal of neurosurgery spine. Vol. 2. 3rd ed; 2005. p. 289-97.

\section{Submit your next manuscript to BioMed Central and we will help you at every step:}

- We accept pre-submission inquiries

- Our selector tool helps you to find the most relevant journal

- We provide round the clock customer support

- Convenient online submission

- Thorough peer review

- Inclusion in PubMed and all major indexing services

- Maximum visibility for your research

Submit your manuscript at www.biomedcentral.com/submit 